\section{The First Total Synthesis of Racemic Hydroxymoloka'iamine}

\author{
Nisar Ullah \\ Chemistry Department, King Fahd University of Petroleum \\ and Minerals, Dhahran 31261, Saudi Arabia \\ Reprint requests to Professor Nisar Ullah. \\ Fax: + 96638604277 . E-mail: nullah@kfupm.edu.sa \\ Z. Naturforsch. 2009, 64b, 879-882; \\ received April 4, 2009 \\ The first total synthesis of racemic hydroxymoloka'iam- \\ ine, a new brominated metabolite isolated from Red Sea \\ sponge Pseudoceratna arabica, was achieved in a high-yield \\ reaction sequence.
}

Key words: Total Synthesis, Natural Product, Bromination

\section{Introduction}

The enormous promise that the sea holds to provide potential lead pharmaceuticals has attracted great interest from the scientific community in the recent years. In an effort to isolate bio-active ingredients from marine sources, investigation of the Red Sea sponge Pseudoceratna arabica has led to the isolation and structure elucidation of the new brominated metabolite hydroxymoloka'iamine (1) [1]. This compound features a bromotyramine skeleton and has structural resemblance with the known metabolite moloka'iamine [2]. The evaluation of the cytotoxic activity against human colon tumor cells (HCT-116) of $\mathbf{1}$ has demonstrated that it was not toxic at a test dose of $10 \mu \mathrm{g} \mathrm{mL}^{-1}$, and such discoveries have important implications for further biological investigations of this class of compounds [1]. As a part of our ongoing research in the total synthesis of bio-active natural products $[3,4]$, we now wish to report a facile synthesis of racemic hydroxymoloka'iamine (1).

\section{Results and Discussion}

The synthesis of hydroxylmoloka'iamine commenced with the bromination of the 4-hydroxyacetophenone with bromine in acetic acid to get the known hydroxyacetophenone dibromide 3 in $93 \%$ yield [5]. The use of sodium acetate as base proved to be effective to improve the yield. The O-alkylation of the latter with an excess of 1,3-dibromopropane in DMF using potassium carbonate as base afforded the desired tribromide 4 in very high yield $(97 \%)$. The subsequent reaction of $\mathbf{4}$ with a stoichiometric amount of trimethylphenylammonium tribromide in THF at $0{ }^{\circ} \mathrm{C}$ to r.t. was unclean and gave the desired $\alpha$-bromoketone $\mathbf{5}$ in $60 \%$ yield along with a significant amount of unwanted side products. However, the problem was overcome by the addition of trimethylphenylammonium tribromide in portions to a solution of tribromide 4 in $\mathrm{THF}$ at $-15{ }^{\circ} \mathrm{C}$ and aging the reaction overnight at $0{ }^{\circ} \mathrm{C}$, which rendered the desired $\alpha$-bromoketone 5 in high yield $(90 \%)$. At this stage we focused on displacement of the two bromo substituents with azide. To this end, sodium azide was added to a stirred solution of $\alpha$-bromoketone 5 in a mixture of ethanol and THF $(4: 1)$. Stirring the mixture first at r. t. followed by heating at $60{ }^{\circ} \mathrm{C}$ afforded a low yield of the desired diazide $6(23 \%)$ along with a few side products which made column chromatography purifications very tedious. In a second attempt the reaction was tried in DMF while the mixture was stirred at $50{ }^{\circ} \mathrm{C}$ overnight which gave the desired product 6 in an acceptable yield (49\%). The reduction of the azido group to the amine with triphenylphosphine followed by crystallization to the PTSA salt yielded the aminoketone 7 in $70 \%$ yield [6], which in turn was subjected to reduction with sodium borohydride at $0{ }^{\circ} \mathrm{C}$ in a mixture of THF and water to afford the target hydroxylmoloka'iamine in an over all yield of $14 \%$ from 5 (Scheme 1).

Because of the difficulty in purification by the column chromatography of the diazide $\mathbf{6}$, coupled with the low overall yield in the synthesis of $\mathbf{1}$ from $\mathbf{5}$, we envisioned that reducing the $\alpha$-bromoketone $\mathbf{5}$ to the $\alpha$-bromoalcohol, and condensation with ammonia, should give the desired target compound $\mathbf{1}$. Thus, ketone 5 was reduced with sodium borohydride at $0{ }^{\circ} \mathrm{C}$ in a mixture of THF and water to get the $\alpha$-bromoalcohol 8, which was treated with an excess of ammonium hydroxide at $55^{\circ} \mathrm{C}$ for $12 \mathrm{~h}$ to afford the target compound 1 in an excellent overall yield (54\% from 5) (Scheme 2). The spectral data of our synthetic 1 coincided with those of the natural material [1].

\section{Conclusion}

In conclusion we have accomplished the first synthesis of racemic hydroxylmoloka'iamine (1) in an overall high-yield reaction sequence. 

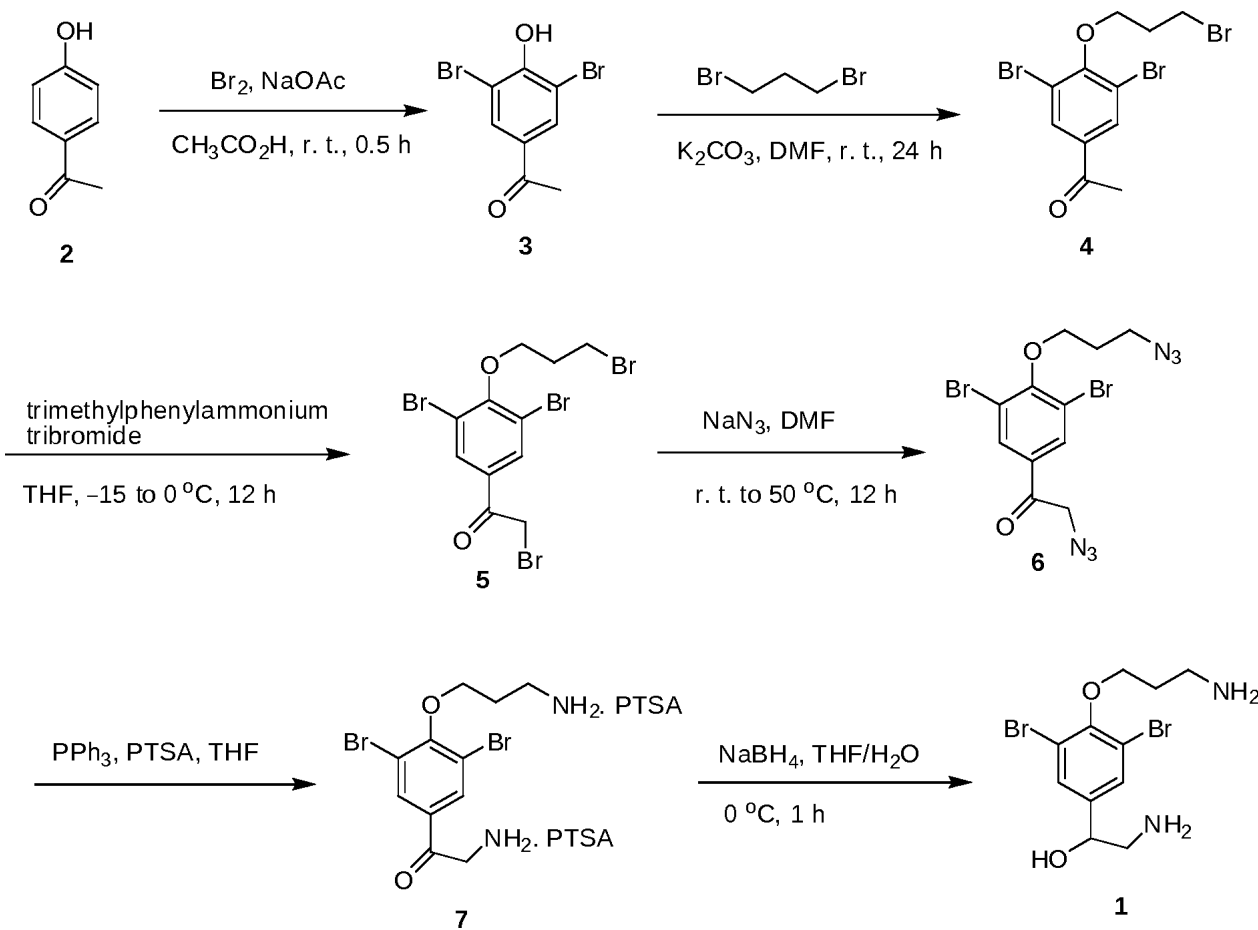

Scheme 1. Synthesis of hydroxylmoloka'iamine (1) via the diazide intermediate 6.<smiles>O=C(CBr)c1cc(Br)c(OCCCBr)c(Br)c1</smiles>

5

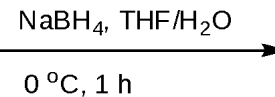

$0^{\circ} \mathrm{C}, 1 \mathrm{~h}$

Scheme 2. Synthesis of hydroxylmoloka'iamine (1) via the bromoalcohol intermediate 8 .

\section{Experimental Section}

\section{1-(3,5-Dibromo-4-hydroxyphenyl)ethanone (3)}

Bromine $(3.02 \mathrm{~g}, 19 \mathrm{mmol})$ in acetic acid $(3 \mathrm{~mL})$ was added dropwise to a mixture of 4-hydroxyacetophenone $(1.22 \mathrm{~g}, 9 \mathrm{mmol})$ and sodium acetate $(2.29 \mathrm{~g}, 27.9 \mathrm{mmol})$ in acetic acid $(20 \mathrm{~mL})$ at r.t. over $10 \mathrm{~min}$. The reaction mixture was stirred for $0.5 \mathrm{~h}$ at r.t., $\mathrm{H}_{2} \mathrm{O}(20 \mathrm{~mL})$ was added, and the solid was filtered, washed with $\mathrm{H}_{2} \mathrm{O}$ and dried under high vacuum to afford the product as a brown solid $(2.46 \mathrm{~g}, 93 \%)$. The physical and spectral data of $\mathbf{3}$ were identical with those of the literature [5].

\section{1-(3,5-Dibromo-4(3-bromopropoxy)phenyl)ethanone (4)}

To a solution of compound 3 (2 g, $6.8 \mathrm{mmol})$ in DMF $(20 \mathrm{~mL})$ at 5 to $10{ }^{\circ} \mathrm{C}$ was added potassium carbonate
$(1.87 \mathrm{~g}, 13.6 \mathrm{mmol})$ in portions, followed by the addition of 1,3-dibromopropane (5.49 g, $27.2 \mathrm{mmol})$, and the mixture was stirred at $\mathrm{r}$.t. for $24 \mathrm{~h}$. The reaction mixture was taken in ethyl acetate $(100 \mathrm{~mL})$ and washed successively with $\mathrm{H}_{2} \mathrm{O}(25 \mathrm{~mL}), 1 \mathrm{~N} \mathrm{HCl}(2 \times 20 \mathrm{~mL})$, sat. $\mathrm{NaHCO}_{3}$ $(20 \mathrm{~mL})$, and brine $(15 \mathrm{~mL})$, and dried over sodium sulfate and evaporated. Column chromatography of the light-yellow oily material, eluting with ethyl acetate: hexanes $(1: 9)$ and then changing to $(2: 8)$ gave the product as a light-orange thick oil (2.74 g, $97 \%)$. - IR (neat): $v=3030,2890,1692$, $1535,1460,1365,1265,1180,1010,910,735,630 \mathrm{~cm}^{-1}$. ${ }^{1} \mathrm{H}$ NMR (500 MHz, $\left.\mathrm{CDCl}_{3}\right): \delta=2.36\left(\mathrm{~m}, 2 \mathrm{H}, 2^{\prime \prime}-\mathrm{H}\right), 2.57$ (s, $\left.3 \mathrm{H}, \mathrm{COCH}_{3}\right), 3.73\left(\mathrm{t}, 2 \mathrm{H}, J=6.7 \mathrm{~Hz}, 3^{\prime \prime}-\mathrm{H}\right), 4.20$ (t, $\left.2 \mathrm{H}, J=5.8 \mathrm{~Hz}, 1^{\prime \prime}-\mathrm{H}\right), 8.10\left(\mathrm{~s}, 2 \mathrm{H}, 2^{\prime}-\mathrm{H}, 6^{\prime}-\mathrm{H}\right)$. ${ }^{13} \mathrm{C} \mathrm{NMR}\left(125.7 \mathrm{MHz}, \mathrm{CDCl}_{3}\right): \delta=26.8\left(\mathrm{COCH}_{3}\right), 29.7$ ( $\left.2^{\prime \prime}-\mathrm{C}\right), 33.3$ (3"-C), 71.0 (1" $\left.{ }^{\prime \prime}-\mathrm{C}\right), 118.4$ (3'-C, 5'-C), 132.9 (2'-C, 6'-C), 135.5 (1'-C), 157.0 (4'-C), 194.2 (CO). - MS 
$(70 \mathrm{eV}): \mathrm{m} / \mathrm{z}(\%)=414(24), 317(14), 290(10), 279$ (100). $-\mathrm{C}_{11} \mathrm{H}_{11} \mathrm{Br}_{3} \mathrm{O}_{2}$ (414.92): calcd. C 31.84, H 2.67; found $\mathrm{C} 31.81, \mathrm{H} 2.70$.

\section{2-Bromo-1-(3,5-dibromo-4-(3-bromopropoxy)phenyl)- ethanone (5)}

To a solution of compound 4 ( $2 \mathrm{~g}, 4.82 \mathrm{mmol})$ in THF $(30 \mathrm{~mL})$ at $-15{ }^{\circ} \mathrm{C}$ was added trimethylphenylammonium tribromide $(1.9 \mathrm{~g}, 5.1 \mathrm{mmol})$, and the mixture was stirred at $0{ }^{\circ} \mathrm{C}$ for $12 \mathrm{~h}$. The solid was filtered, and to the filtrate was added ethyl acetate $(50 \mathrm{~mL})$. The mixture was washed successively with $\mathrm{H}_{2} \mathrm{O}(20 \mathrm{~mL})$ and brine $(15 \mathrm{~mL})$, and the organic layer was dried over $\mathrm{Na}_{2} \mathrm{SO}_{4}$ and evaporated to leave a dark-orange oil, which was resolved on a silica column eluting with ethyl acetate : hexanes $(1: 9)$ to get the product as an off-white crystalline solid $(2.14$ g, $90 \%)$. M. p. $67-68^{\circ} \mathrm{C}$. IR (neat): $v=3050,2937,2890,1698,1540,1451,1372$, 1280, 1177, 1004, 907, 853, 734, $630 \mathrm{~cm}^{-1} .-{ }^{1} \mathrm{H} \mathrm{NMR}$ $\left(500 \mathrm{MHz}, \mathrm{CDCl}_{3}\right): \delta=2.43\left(\mathrm{~m}, 2 \mathrm{H}, 2^{\prime \prime}-\mathrm{H}\right), 3.73(\mathrm{t}, 2 \mathrm{H}, J=$ $\left.6.4 \mathrm{~Hz}, 3^{\prime \prime}-\mathrm{H}\right), 4.22$ (t, $\left.2 \mathrm{H}, J=5.5 \mathrm{~Hz}, 1^{\prime \prime}-\mathrm{H}\right), 4.38$ (s, $2 \mathrm{H}$, 2-H), 8.12 (s, $\left.2 \mathrm{H}, 2^{\prime}-\mathrm{H}, \mathrm{H}-6^{\prime}-\mathrm{H}\right) .-{ }^{13} \mathrm{C}$ NMR (125.7 MHz, $\left.\mathrm{CDCl}_{3}\right): \delta=29.6\left(2^{\prime \prime}-\mathrm{C}\right), 30.0(2-\mathrm{C}), 33.2\left(3^{\prime \prime}-\mathrm{C}\right), 71.1$ $\left(1^{\prime \prime}-\mathrm{C}\right), 118.9\left(3^{\prime}-\mathrm{C}, 5^{\prime}-\mathrm{C}\right), 131.8\left(2^{\prime}-\mathrm{C}, 6^{\prime}-\mathrm{C}\right), 135.4\left(1^{\prime}-\mathrm{C}\right)$, $157.3\left(4^{\prime}-\mathrm{C}\right), 188.0(\mathrm{CO}) .-\mathrm{MS}(70 \mathrm{eV}): \mathrm{m} / \mathrm{z}(\%)=494$ (20), 399 (26), 372 (9), 279 (100), 121 (20). - $\mathrm{C}_{11} \mathrm{H}_{10} \mathrm{Br}_{4} \mathrm{O}_{2}$ (493.81): calcd. C 26.75, H 2.04; found C 26.73, H 2.06.

\section{2-Azido-1-(4-(3-azidopropoxy)-3,5-dibromophenyl)- ethanone (6)}

To a solution of compound 5 ( $1 \mathrm{~g}, 2.02 \mathrm{mmol})$ in DMF $(15 \mathrm{~mL})$ was added sodium azide $(0.27 \mathrm{~g}, 4.14 \mathrm{mmol})$ in one portion, and the mixture was heated at $50{ }^{\circ} \mathrm{C}$ for $12 \mathrm{~h}$. After addition of $\mathrm{H}_{2} \mathrm{O}(10 \mathrm{~mL})$, the reaction mixture was extracted with ethyl acetate $(25 \mathrm{~mL})$. The organic layer was washed successively with $1 \mathrm{~N} \mathrm{HCl}(2 \times, 10 \mathrm{~mL})$, sat. $\mathrm{NaHCO}_{3}$ $(15 \mathrm{~mL})$ and brine $(10 \mathrm{~mL})$, dried over sodium sulfate and evaporated. Column chromatography of the dark-orange oily material eluting with ethyl acetate: hexanes (1:9) gave the product as a dark-orange thick oil $(0.41 \mathrm{~g}, 49 \%)$. - IR (neat): $v=3073,2954,2197,2104,1698,1580,1539,1452,1379$, 1250, 1000, 919, $737 \mathrm{~cm}^{-1} .-{ }^{1} \mathrm{H}$ NMR (500 MHz, $\left.\mathrm{CDCl}_{3}\right)$ : $\delta=2.45\left(\mathrm{~m}, 2 \mathrm{H}, 2^{\prime \prime}-\mathrm{H}\right), 3.83\left(\mathrm{t}, 2 \mathrm{H}, J=6.3 \mathrm{~Hz}, 3^{\prime \prime}-\mathrm{H}\right)$, $4.23\left(\mathrm{t}, 2 \mathrm{H}, J=5.6 \mathrm{~Hz}, 1^{\prime \prime}-\mathrm{H}\right), 4.49$ (s, $\left.2 \mathrm{H}, 2-\mathrm{H}\right), 8.05$ (s, $\left.2 \mathrm{H}, 2^{\prime}-\mathrm{H}, 6^{\prime}-\mathrm{H}\right) .-{ }^{13} \mathrm{C} \mathrm{NMR}\left(125.7 \mathrm{MHz}, \mathrm{CDCl}_{3}\right): \delta=30.0$ $\left(2^{\prime \prime}-\mathrm{C}\right), 48.7\left(3^{\prime \prime}-\mathrm{C}\right), 54.8(2-\mathrm{C}), 71.2\left(1^{\prime \prime}-\mathrm{C}\right), 118.8\left(3^{\prime}-\mathrm{C}\right.$, $\left.5^{\prime}-\mathrm{C}\right), 131.9\left(2^{\prime}-\mathrm{C}, 6^{\prime}-\mathrm{C}\right), 135.4\left(1^{\prime}-\mathrm{C}\right), 157.4\left(4^{\prime}-\mathrm{C}\right), 195.2$ (CO). - MS (70 eV): $\mathrm{m} / z(\%)=418$ (3), 362 (18), 279 (100). $-\mathrm{C}_{11} \mathrm{H}_{10} \mathrm{Br}_{2} \mathrm{~N}_{6} \mathrm{O}_{2}$ (418.04): calcd. C 31.60, H 2.41, N 20.10; found C 31.56, H 2.46, N 20.03.

2-Amino-1-(4-(3-aminopropoxy)-3,5-dibromophenyl)ethanone p-toluenesulfonic acid salt (7)

To a solution of compound $6(0.35 \mathrm{~g}, 0.84 \mathrm{mmol})$ in THF $(10 \mathrm{~mL})$ was added triphenylphosphine $(0.22 \mathrm{~g}, 0.84 \mathrm{mmol})$, and when the solution was homogeneous, PTSA (0.8 g, $4.2 \mathrm{mmol}$ ) was added in small portions. The reaction mixture was stirred for $24 \mathrm{~h}$. The resulting solid was filtered and allowed to dry under vacuum to get the product as an off-white solid $(0.42 \mathrm{~g}, 70 \%)$. M. p. $264-265{ }^{\circ} \mathrm{C}$. - IR (neat): $v=3070,2880,2010,1690,1590,1545,1451,1370$, 1270, 1170, 1003, 910, $740 \mathrm{~cm}^{-1} .-{ }^{1} \mathrm{HNMR}(500 \mathrm{MHz}$, [D 6 DMSO): $\delta=2.26$ (s, $\left.3 \mathrm{H}, \mathrm{CH}_{3}\right), 2.27\left(\mathrm{~s}, 3 \mathrm{H}, \mathrm{CH}_{3}\right)$, $2.44\left(\mathrm{~m}, 2 \mathrm{H}, 2^{\prime \prime}-\mathrm{H}\right), 3.88\left(\mathrm{t}, 2 \mathrm{H}, J=6.4 \mathrm{~Hz}, 3^{\prime \prime}-\mathrm{H}\right), 4.22(\mathrm{t}$, $\left.2 \mathrm{H}, J=5.5 \mathrm{~Hz}, 1^{\prime \prime}-\mathrm{H}\right), 4.52$ (s, $\left.2 \mathrm{H}, 2-\mathrm{H}\right), 7.12$ (d, $4 \mathrm{H}, J=$ $8.0 \mathrm{~Hz}$, aromatic-H), $7.48(\mathrm{~d}, 4 \mathrm{H}, J=8.0 \mathrm{~Hz}$, aromatic-H), 8.06 (s, $\left.2 \mathrm{H}, \mathrm{H}-2^{\prime}, 6^{\prime}-\mathrm{H}\right), 8.10$ (broad s, $3 \mathrm{H}$ ), 8.14 (broad s, $3 \mathrm{H}) .-{ }^{13} \mathrm{C}$ NMR $\left(125.7 \mathrm{MHz},\left[\mathrm{D}_{6}\right] \mathrm{DMSO}\right): \delta=20.7$ $\left(\mathrm{CH}_{3}\right), 29.9\left(2^{\prime \prime}-\mathrm{C}\right), 45.1\left(3^{\prime \prime}-\mathrm{C}\right), 46.2(2-\mathrm{C}), 71.1\left(1^{\prime \prime}-\mathrm{C}\right)$, 118.8 ( $\left.3^{\prime}-\mathrm{C}, 5^{\prime}-\mathrm{C}\right), 125.6$ (aromatic-C), 128.2 (aromatic-C), 131.8 (2'-C, $\left.6^{\prime}-\mathrm{C}\right), 135.5$ (aromatic-C), 137.8 (1'-C), 144.9 (aromatic-C), 157.3 (4'-C), $191.4(\mathrm{CO}) .-\mathrm{MS}(70 \mathrm{eV}): \mathrm{m} / \mathrm{z}$ $(\%)=366$ (12), 308 (16), 279 (100).

\section{2-Bromo-1-(3,5-dibromo-4-(3-bromopropoxy)phenyl)- ethanol (8)}

To a solution of compound $5(0.2 \mathrm{~g}, 0.40 \mathrm{mmol})$ in a mixture of THF and $\mathrm{H}_{2} \mathrm{O}(4: 2,6 \mathrm{~mL})$ at $0{ }^{\circ} \mathrm{C}$ was added sodium borohydride $(0.019 \mathrm{~g}, 0.5 \mathrm{mmol})$ and after being stirred for $0.5 \mathrm{~h}, \mathrm{H}_{2} \mathrm{O}(5 \mathrm{~mL})$ was added. After extraction of the mixture with ethyl acetate $(2 \times 15 \mathrm{~mL})$, the organic layer was washed with brine $(5 \mathrm{~mL})$, dried over sodium sulfate and evaporated. Column chromatography of the colorless oily material eluting with ethyl acetate: hexanes (1:9) gave the product as a colorless thick oil $(0.18 \mathrm{~g}, 90 \%)$. - IR (neat): $v=3325,3030$, 2937, 2890, 1545, 1450, 1370, 1281, 1175, 1003, 905, 853, $735,630 \mathrm{~cm}^{-1} .-{ }^{1} \mathrm{H}$ NMR $\left(500 \mathrm{MHz}, \mathrm{CDCl}_{3}\right): \delta=2.40$ (m, $\left.2 \mathrm{H}, 2^{\prime \prime}-\mathrm{H}\right), 2.69$ (d, $\left.1 \mathrm{H}, J=3.1 \mathrm{~Hz}, \mathrm{OH}\right), 3.48(\mathrm{dd}, 1$ $\mathrm{H}, J=10.6,8.8 \mathrm{~Hz}, 2-\mathrm{H}), 3.60(\mathrm{dd}, 1 \mathrm{H}, J=10.3,3.3 \mathrm{~Hz}$, $2-\mathrm{H}), 3.73\left(\mathrm{t}, 2 \mathrm{H}, J=6.4 \mathrm{~Hz}, 3^{\prime \prime}-\mathrm{H}\right), 4.13(\mathrm{t}, 2 \mathrm{H}, J=5.8 \mathrm{~Hz}$, $\left.1^{\prime \prime}-\mathrm{H}\right), 4.86(\mathrm{dd}, 1 \mathrm{H}, J=8.8,3.3 \mathrm{~Hz}, 1-\mathrm{H}), 7.55$ (s, $2 \mathrm{H}, 2^{\prime}-\mathrm{H}$, $\left.6^{\prime}-\mathrm{H}\right) .-{ }^{13} \mathrm{C}$ NMR $\left(125.7 \mathrm{MHz}, \mathrm{CDCl}_{3}\right): \delta=29.9\left(2^{\prime \prime}-\mathrm{C}\right)$, 33.4 (3" $\left.{ }^{\prime \prime}-\mathrm{C}\right), 39.5$ (2-C), $70.8\left(1^{\prime \prime}-\mathrm{C}\right), 72.1$ (1-C), 118.5 (3'-C, $\left.5^{\prime}-\mathrm{C}\right), 130.3\left(2^{\prime}-\mathrm{C}, 6^{\prime}-\mathrm{C}\right), 138.8\left(1^{\prime}-\mathrm{C}\right), 152.8\left(4^{\prime}-\mathrm{C}\right) .-\mathrm{MS}$ $(70 \mathrm{eV}): \mathrm{m} / \mathrm{z}(\%)=496(8), 416(11), 398(19), 335$ (9), 296 (18), 278 (100), 215 (50). $-\mathrm{C}_{11} \mathrm{H}_{12} \mathrm{Br}_{4} \mathrm{O}_{2}$ (495.83): calcd. C 26.65, H 2.44; found C 26.63, H 2.46.

\section{Hydroxylmoloka'iamine (1)}

From compound 7: Sodium borohydride (0.130 g, 3.36 mmol) was added to a solution of compound $7(0.4 \mathrm{~g}$, $0.56 \mathrm{mmol})$ in a mixture of THF and $\mathrm{H}_{2} \mathrm{O}(4: 2,6 \mathrm{~mL})$ at $0{ }^{\circ} \mathrm{C}$, and the reaction mixture was stirred for $1 \mathrm{~h}$ at this temperature. The $\mathrm{pH}$ of the mixture was adjusted to 9 by $1 \mathrm{~N} \mathrm{NaOH}$, the mixture was extracted with ethyl acetate $(2 \times 10 \mathrm{~mL})$, and the organic layer was washed with brine $(5 \mathrm{~mL})$, dried over sodium sulfate and evaporated to get the product as a colorless thick oil, which was purified on a silica 
column. Elution with $\mathrm{MeOH}: \mathrm{CHCl}_{3}(5: 95)$, then changing to $25: 75$, gave the product as a white solid $(0.082 \mathrm{~g}, 40 \%)$. The physical and spectral data of our synthetic 1 were identical with those of the natural material [1].

From compound 8: To a solution of compound $8(0.16 \mathrm{~g}$, $0.32 \mathrm{mmol})$ in THF $(1 \mathrm{~mL})$ was added aqueous ammonium hydroxide $(3 \mathrm{~mL})$. The reaction mixture was stirred at $55{ }^{\circ} \mathrm{C}$ for $12 \mathrm{~h}$, then extracted with ethyl acetate $(2 \times 10 \mathrm{~mL})$, and the organic layer was washed with brine $(5 \mathrm{~mL})$, dried over sodium sulfate, evaporated, and purified on a column as described for compound $\mathbf{7}$ to get the desired $\mathbf{1}$ $(0.07 \mathrm{~g}, 60 \%)$.
[1] J. M. Badr, L. A. Shaala, M. I. Abou-Shoer, M. K. Tawfik, A-A. M. Habib, J. Nat. Prod. 2008, 71, $1472-$ 1476.

[2] M. T. Hamann, P. J. Scheuer, M. Kelly-Borges, J. Org. Chem. 1993, 58, 6565-6569.

[3] N. Ullah, M. A. Khaled, Tetrahedron Lett. 2009, 50, $158-160$

[4] N. Ullah, Z. Naturforsch. 2009, 64b, 464-466.
[5] U. V. Mallavadhani, A. Mahapatra, K. Narasimhan, L. D. Sahoo, Indian J. Chem., Section B: Organic Chemistry Including Medicinal Chemistry 2002, $41 B(7), 1460-1466$.

[6] J. T. Gupton, R. B. Miller, K. E. Krumpe, S. C. Clough, E. J. Banner, R.P.F. Kanters, K.X. Du, K. M. Keertikar, N.E. Lauerman, J.M. Solano, B. R. Adams, D. W. Callahan, B. A. Little, A. B. Scharfa, J. A. Sikorskid, Tetrahedron 2005, 61, 1845-1854. 